\title{
AN ISOPERIMETRIC INEQUALITY FOR ARTIN GROUPS OF FINITE TYPE
}

\author{
KAY TATSUOKA
}

\begin{abstract}
We show that Artin groups of finite type satisfy a quadratic isoperimetric inequality. Moreover we describe an explicit algorithm to solve the word problem in quadratic time.
\end{abstract}

\section{INTRODUCTION}

Let $G$ be a group with presentation $\langle C ; R\rangle$, where $C \subset G$ is a set of generators and $R$ a set of defining relations. Let $F(C)$ be the free group freely generated by $C$ and let $N(R)$ denote the kernel of the epimorphism from $F(C)$ onto $G$. Given $f \in F(C)$ we can define the length $|f|$ to be the word length of $f$ written as a word in $C$. If $f \in N(R)$ one can also define the combinatorial area $A(f)$ ([Gr] or [CEHPT]). Essentially $A(f)$ is the minimal number of substitutions in the defining relators required to shorten $f$ to the identity element.

A group satisfies a linear isoperimetric inequality if there exists a constant $k>1$ such that $A(f) \leq k \cdot|f|$ for all $f \in N(R)$. Hyperbolic groups, in the sense of Gromov, satisfy a linear isoperimetric inequality.

A group satisfies a quadratic isoperimetric inequality if there exists a constant $k>1$ such that $A(f) \leq k \cdot|f|^{2}$ for all $f \in N(R)$. A group satisfying a quadratic isoperimetric inequality, but not a linear one, is $Z+Z$.

The notion of an automatic group was introduced by Thurston and developed by Cannon, Epstein, and others. These groups satisfy a quadratic isoperimetric inequality [CEHPT]. Thurston has shown that the braid groups, an important special case of Artin groups of finite type, are automatic [Th]. The reader interested in automatic groups is referred to that paper. Charney [Ch] has generalized this result to Artin groups of finite type. In this paper we directly verify that Artin groups of finite type, defined below, satisfy a quadratic isoperimetric inequality. Moreover our proof gives a solution to the word problem in quadratic time. The latter result appears to be new.

Given a group $G$ with generating set $C$ define for $x, y \in C$ and $m \in Z^{+}$ $\operatorname{prod}(x, y, m) \in F(C)$ to be the alternating word $x y x y \ldots$ of length $m$. A Coxeter group $W$ has generating set $S$ and relations

$$
s^{2}=1 \text { for each } s \in S,
$$

Received by the editors April 15, 1991.

1991 Mathematics Subject Classification. Primary 20F10, 20 F36.

Key words and phrases. Artin group, braid group, isoperimetric inequality, word problem. 


$$
\begin{aligned}
& \operatorname{prod}\left(s_{i}, s_{j}, m_{i j}\right)=\operatorname{prod}\left(s_{j}, s_{i}, m_{j i}\right) \\
& \qquad \text { for certain } s_{i}, s_{j} \in S, m_{i j}=m_{j i} \in Z^{+} .
\end{aligned}
$$

The associated Artin group $\widetilde{W}$ has generating set $\widetilde{S}$ in a fixed one-to-one correspondence with $S$. The relations for $\widetilde{W}$ are obtained from the relations for $W$ by omitting the $s^{2}=1$ relations and using the correspondence between $\widetilde{S}$ and $S$ to rewrite the other relations. $\widetilde{W}$ has relations

$$
\begin{aligned}
& \operatorname{prod}\left(\sigma_{i}, \sigma_{j}, m_{i j}\right)=\operatorname{prod}\left(\sigma_{j}, \sigma_{i}, m_{j i}\right) \\
& \qquad \text { for certain } \sigma_{i}, \sigma_{j} \in \widetilde{S}, m_{i j}=m_{j i} \in Z^{+} .
\end{aligned}
$$

When $W$ is finite $\widetilde{W}$ is an Artin group of finite type. When $W$ is the symmetric group on $n$ letters $\widetilde{W}$ is the braid group $B_{n+1}$. We state formally the main theorems.

Theorem A. Let $\widetilde{W}$ be an Artin group of finite type. Then $\widetilde{W}$ satisfies a quadratic isoperimetric inequality.

Theorem B. Let $\widetilde{W}$ be an Artin group of finite type. Then the word problem for $\widetilde{W}$ is solvable in quadratic time.

We state Theorem B more precisely in $\S 4$. For now it means given $f \in F(\widetilde{S})$ roughly $|f|^{2}$ substitutions are needed to decide if $f$ represents the identity. We emphasize that the algorithm is explicit. Theorem $B$ is especially interesting when $\widetilde{W}$ is the braid group. Since the elements of the braid group are closely related to links in $S^{3}$ [Bi], Theorem B makes more efficient the process of distinguishing links.

The paper is organized as follows. In $\S 2$ we summarize some standard facts about finite Coxeter groups. These facts are used to define a cell complex $\widetilde{K}$. The 2-skeleton of $\widetilde{K}$ is the Cayley complex for $\widetilde{W}$. This cell complex is proved to be contractible in [T]; however we use only the fact that $\widetilde{K}$ is simply connected. In $\S 3$ we summarize the work of Garside, Brieskorn-Saito, and Deligne concerning positive words in $\widetilde{W}$. We also prove the key proposition. In $\S 4$ we prove Theorems A and B by shortening edge paths in $\widetilde{K}$. We give examples of the algorithm.

Notation 1.4. Let $C$ be a set of letters, $F(C)$ the set of words in $C, \Gamma$ a complex with edges labelled with $c \in C$. Let $v$ be a vertex of $\Gamma$. Suppose $g \in F(C)$ labels an edge path in $\Gamma$ starting at $v$. Define $[g]_{v}$ to be this edge path. Define $v \cdot g$ to be the terminal vertex of $[g]_{v}$.

\section{THE COXETER COMPLEX AND ITS DUAL}

From now on $W$ is a finite Coxeter group. Then $W$ has a well-known description as a finite reflection group. Let $V$ be an $n$-dimensional real vector space, $\mathscr{H}$ a finite set of hyperplanes through the origin. Let $W$ be the finite reflection group generated by reflections $s_{H}$ for $H \in \mathscr{H}$. Assume no nonzero vector of $V$ is fixed by $W$. The Coxeter complex $\Sigma$ is the abstract simplicial complex with geometric realization the unit sphere $S^{n-1}$ equipped with triangulation cut out by $\mathscr{H}$. A chamber is a maximal dimensional open simplex of $\Sigma$. A codim 1 face of the closure of a chamber is supported by a hyperplane. Each hyperplane is called a wall.

We refer the reader to $[\mathrm{Br}]$ for proofs of the following standard facts. 
Lemma 2.1. Let $S$ denote the set of reflections in the walls of a fixed chamber of $\Sigma$. Then $(a) S$ generates $W(b)$ and the elements of $S$ satisfy relations (1.1), (1.2).

Let $w \in W$ and let $s_{1} s_{2} \cdots s_{k} \in F(S)$ represent $w$. We say the word $s_{1} s_{2} \cdots s_{k}$ is geodesic in $S$ if no shorter word of $F(S)$ represents $w$. Given $w, w^{\prime} \in W$ define $d\left(w, w^{\prime}\right)$ to be the length of a geodesic word of $F(S)$ representing $w^{-1} w^{\prime}$. Given a subset $A$ of $W$ and $w \in W$ define $d(w, A)=$ $\inf _{w^{\prime} \in A} d\left(w, w^{\prime}\right)$. Here $e$ is the identity element of $W$.

Lemma 2.2. Let $w \in W, s \in S$. Then $d(w s, e)=d(w, e)+1$ or $d(w, e)-1$.

Lemma 2.3. There is a unique element $w_{M} \in W$ such that $d\left(e, w_{M}\right)$ is maximal. Further, let $s_{1} s_{2} \cdots s_{k} \in F(S)$ be a geodesic in $S$ representing $w \in$ $W$. Let $s_{k+1} \cdots s_{m} \in F(S)$ be geodesic in $S$ representing $w^{-1} w_{M}$. Then $s_{1} s_{2} \cdots s_{k+1} \cdots s_{m} \in F(S)$ is a geodesic word in $S$ representing $w_{M}$.

A special subgroup $W^{\prime}$ of $W$ is one generated by a subset $S^{\prime}$ of $S$. We write $W^{\prime}=\left\langle S^{\prime}\right\rangle$. By convention $\langle e\rangle$ is a special subgroup. A special coset is a left coset of a special subgroup.

Lemma 2.4. Let $w W^{\prime}$ be a special coset, where $W^{\prime}=\left\langle S^{\prime}\right\rangle$ for $S^{\prime} \subset S$. Then there is a unique element $w_{0} \in w W^{\prime}$ satisfying $d\left(e, w_{0}\right)=d\left(e, w W^{\prime}\right)$. Further, let $s_{1} s_{2} \cdots s_{k} \in F(S)$ be a geodesic in $S$ representing $w_{0}$. Let $s_{k+1} \cdots s_{p} \in$ $F\left(S^{\prime}\right)$ be geodesic in $S^{\prime}$. Then $s_{1} s_{2} \cdots s_{k} s_{k+1} \cdots s_{p} \in F(S)$ is geodesic in $S$.

A poset is a partially ordered set. The set of simplices of a simplicial complex, ordered by the face relation, is a poset. By convention the empty set is included as a simplex. The empty set is given dimension -1 (see [ $\mathrm{Br}$, Chapter 1]). The set special cosets of $W\}^{\text {op }}$, ordered by the opposite of inclusion, is a poset. Note $W$ acts on special cosets of $W$ \} $^{\text {op }}$ by left translation. A map between posets is a poset isomorphism if it is an order-preserving bijection. See Figure 2.1 .

Lemma 2.5. There is a poset isomorphism $\Sigma \approx\{\text { special cosets }\}^{\text {op }}$ which is compatible with the $W$-action.

By Lemma 2.5 the top-dimensional simplices of $\Sigma$, or chambers, are in oneto-one correspondence with the elements of $W$ (the elements of $W$ are precisely the left cosets of the special subgroup $\langle e\rangle)$. The codim 1 faces of $\Sigma$ are in one-to-one correspondence with the special cosets $w\langle s\rangle, w \in W, s \in S$. Write $\Sigma(W)$ for $\Sigma$. Define $\Sigma_{d}(W)$ (or $\Sigma_{d}$ for short) to be the dual Coxeter complex. Note $\Sigma_{d}$ is realized geometrically as an $(n-1)$-sphere. Let $W^{\prime}=\left\langle S^{\prime}\right\rangle$, where $S^{\prime} \subseteq S$ has cardinality $p$. We can similarly define $\Sigma_{d}\left(W^{\prime}\right)$ for $W^{\prime}$ a nontrivial special subgroup of $W . \Sigma_{d}\left(W^{\prime}\right)$ is realized geometrically as a $(p-1)$-sphere.

Definition 2.6. Let $W^{\prime}$ be a special subgroup of $W$. If $W^{\prime}=\langle e\rangle$ define $\widehat{\Sigma}_{d}\left(W^{\prime}\right)$ to be a 0 -cell. If $W^{\prime}=\left\langle S^{\prime}\right\rangle$, where $S^{\prime} \subset S$ has cardinality $p$, define $\widehat{\Sigma}_{d}\left(W^{\prime}\right)$ to be a $p$-cell with $\partial \widehat{\Sigma}_{d}\left(W^{\prime}\right)$ identified with $\Sigma_{d}\left(W^{\prime}\right)$.

We can think of $\widehat{\Sigma}_{d}\left(W^{\prime}\right)$ as the cone over $\Sigma_{d}\left(W^{\prime}\right)$. We can describe the cell structure of $\widehat{\Sigma}_{d}(W)$, hence of $\Sigma_{d}(W)$, in terms of the cells $\widehat{\Sigma}_{d}\left(W^{\prime}\right)$. Let $w W^{\prime}$ be a codim $p$ simplex of $\Sigma(W)$. If $p=0$ then $W^{\prime}=\langle e\rangle$; otherwise $w^{\prime}=\left\langle S^{\prime}\right\rangle$, 

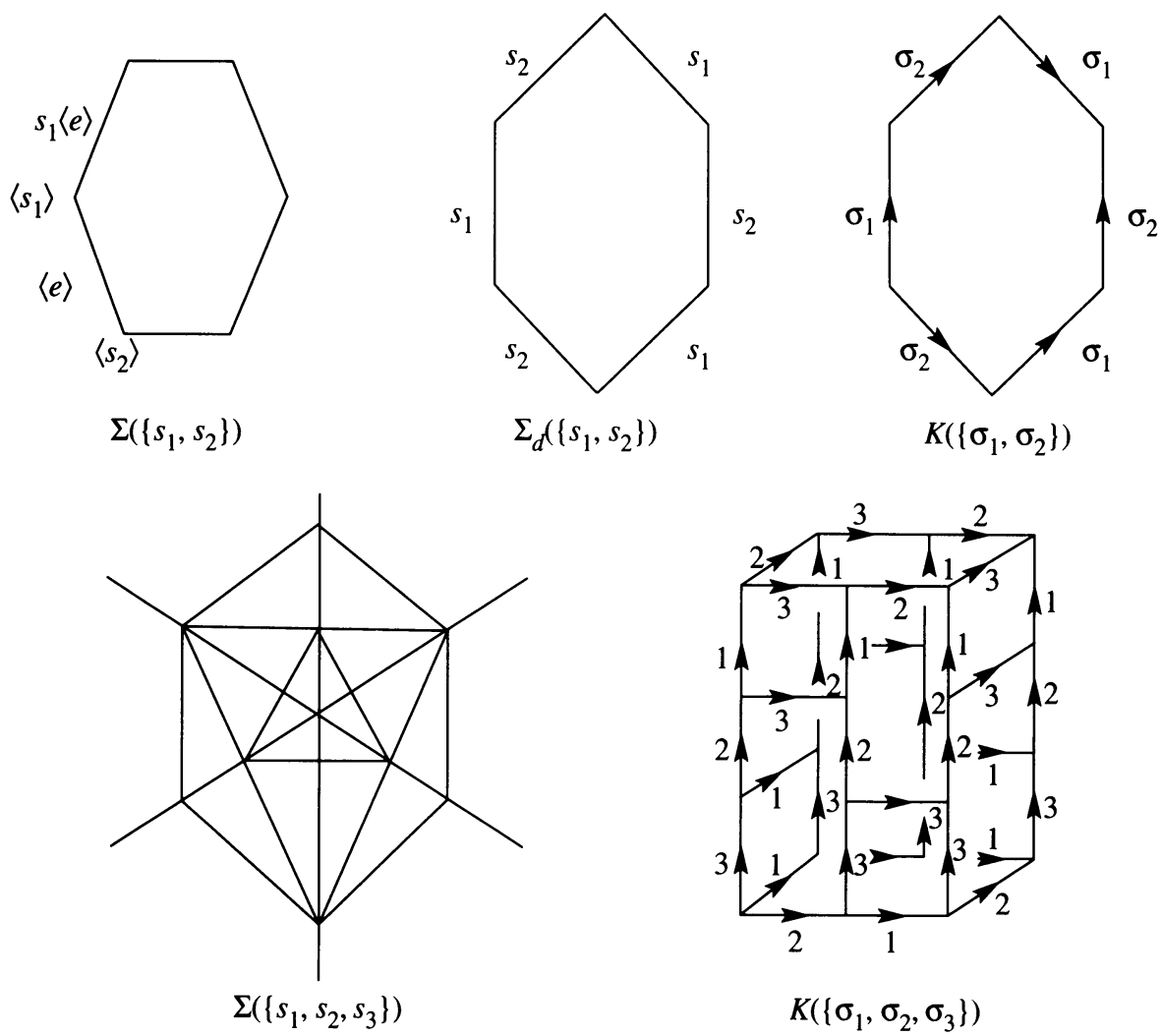

Cells for $B_{3}$ and $B_{4}$.

FIGURe 2.1

where $S^{\prime} \subset S$ has cardinality $p$. We identify the dual $p$-cell of $w W^{\prime}$, which we describe inductively on $p$. Each vertex of $\Sigma_{d}(W)$ is dual to a chamber of $\Sigma(W)$. Identify $\widehat{\Sigma}_{d}(\langle e\rangle)$ with the vertex dual to $\langle e\rangle$. Then each 0 -cell of $\Sigma_{d}(W)$ has the form $w \widehat{\Sigma}_{d}(\langle e\rangle)$. Each edge of $\Sigma_{d}(W)$ is dual to a codim 1 face of $\Sigma(W)$. For each $s \in S$ identify $\widehat{\Sigma}_{d}(\langle s\rangle)$ with the 1-cell dual to $\langle s\rangle$. Then each 1-cell of $\Sigma_{d}(W)$ has the form $w \widehat{\Sigma}_{d}(\langle s\rangle)$. In general let $W^{\prime}=\left\langle S^{\prime}\right\rangle$, where card $S^{\prime}=p$ and $p>1$, be a proper special subgroup of $W$. We claim $\widehat{\Sigma}_{d}\left(W^{\prime}\right)$ can be identified with the $p$-cell of $\Sigma_{d}(W)$ dual to the codim $p \operatorname{simplex} W^{\prime}$ of $\Sigma(W)$.

Given a poset $A$ and $a \in A$ define $A \geq a$ to be the subposet consisting of all elements $\geq a$. Suppose $W^{\prime}=\left\langle S^{\prime}\right\rangle$ is a face of $w\langle T\rangle$, where $w \in W$ and $T \subset S$. Then the dual of $w\langle T\rangle$ is a subcell of the dual of $W^{\prime}$. Since the ordering on $\Sigma$ is reverse inclusion, $w\langle T\rangle \subset W^{\prime}$. This implies $w \in W^{\prime}$ and $T \subset S^{\prime}$. Thus $w\langle T\rangle$ is a special coset of $W^{\prime}$. We conclude

$$
\Sigma_{\geq W^{\prime}}=\left\{\text { special cosets of } W^{\prime}\right\}^{\text {op }} \approx \Sigma\left(W^{\prime}\right) .
$$

We can therefore identify the set of proper subcells of the dual of $W^{\prime}$ with $\Sigma_{d}\left(W^{\prime}\right)$. It follows, as claimed, that the dual of $W^{\prime}$ is precisely $\widehat{\Sigma}_{d}\left(W^{\prime}\right)$. We summarize. 
Lemma 2.7. The cells of $\widehat{\Sigma}_{d}(W)$ are in one-to-one correspondence with special cosets of $W\}$, ordered by inclusion. The correspondence is given by $w \widehat{\Sigma}_{d}\left(W^{\prime}\right) \leftrightarrow$ $w W^{\prime}$.

Label each edge $w \widehat{\Sigma}_{d}(\langle s\rangle)$ of $\widehat{\Sigma}_{d}(W)$ with the letter $s$. Since a word $s_{1} \cdots s_{k}$ $\in F(S)$ labels an edge path in $\widehat{\Sigma}_{d}(W)$ beginning at any vertex of $\widehat{\Sigma}_{d}(W)$, the metric $d$ defined for pairs of elements of $W$ can be identified with the edge path metric on $\widehat{\Sigma}_{d}(W)$. We define an $n$-cell $K(\widetilde{S})$ by putting a new labelling on $\widehat{\Sigma}_{D}(W)$ (see Figure 2.1).

Definition 2.8. Let $\widetilde{S}^{\prime} \subseteq \widetilde{S}$ be a subset of cardinality $p>0$. Let $S^{\prime}$ be the corresponding subset of $S$ and set $W^{\prime}=\left\langle S^{\prime}\right\rangle$. Define $K\left(\widetilde{S}^{\prime}\right)$ to be a $p$-cell with cell decomposition identical to $\widehat{\Sigma}_{d}\left(W^{\prime}\right)$, with edges labelled as follows. Let $w \widehat{\Sigma}_{d}(\langle e\rangle)$ be a vertex of $\widehat{\Sigma}_{d}\left(W^{\prime}\right)$ and let $\left.w \widehat{\Sigma}_{d}(\langle s]\rangle\right), s \in S^{\prime}$, be an attached edge. Let $\sigma$ be the element of $\widetilde{S}^{\prime}$ corresponding to $s$. Label $w \widehat{\Sigma}_{d}(\langle s\rangle)$ with $\sigma$ directed away from $w \widehat{\Sigma}_{d}(\langle e\rangle)$ if $d(w s, e)=d(w, e)+1$ and directed towards $w \widehat{\Sigma}_{d}(\langle e\rangle)$ if $d(w s, e)=d(w, e)-1$ (Lemma 2.2). When $\widetilde{S}^{\prime}=\widetilde{S}$, the $n$-cell $K(\widetilde{S})$ is called the fundamental $n$-cell.

By the definition there is a unique vertex $v_{0}$ of $K\left(\widetilde{S}^{\prime}\right)$ such that each edge attached to $v_{0}$ is directed away from $v_{0}$. We call $v_{0}$ the source vertex of $K\left(\tilde{S}^{\prime}\right)$. By Lemma 2.3 there is a unique vertex $v_{M}$, which we call the sink vertex of $K\left(\tilde{S}^{\prime}\right)$, such that every edge attached to $v_{M}$ is directed towards $v_{M}$.

Since $K(\widetilde{S})$ has the same underlying cell structure as $\widehat{\Sigma}_{d}(W)$, a cell $K^{\prime}$ of $K(\widetilde{S})$ has the underlying cell structure of some $w \widehat{\Sigma}_{d}\left(W^{\prime}\right)$. By Lemma 2.4 there is a unique vertex $w_{0} \widehat{\Sigma}_{d}(\langle e\rangle)$ of $w \widehat{\Sigma}_{d}\left(W^{\prime}\right)$ closest to $\widehat{\Sigma}_{d}(\langle e\rangle)$. Also by Lemma 2.4 the labelling on $w_{0} \widehat{\Sigma}_{d}\left(W^{\prime}\right)$ induced by the labelling of $\widehat{\Sigma}_{d}(W)$ is the same as the labelling of $\widehat{\Sigma}_{d}\left(W^{\prime}\right)$ as described in Definition 2.8. Thus $K^{\prime}$ is a copy of $K\left({\widetilde{S^{\prime}}}^{\prime}\right)$, where $\widetilde{S}^{\prime}$ corresponds to $S^{\prime}$ and $W^{\prime}=\left\langle S^{\prime}\right\rangle$. We summarize.

Lemma 2.9. The cells of $K(\widetilde{S})$ are in one-to-one correspondence with special cosets of $W\}$, ordered by inclusion. The correspondence sends each special coset $w\left\langle S^{\prime}\right\rangle$, where $S^{\prime}$ has cardinality $p>0$, to a copy of $K\left(\tilde{S}^{\prime}\right)$.

Remark 2.10. Each 2-cell of $K(\widetilde{S})$ has the form $K\left(\left\{\sigma_{i}, \sigma_{j}\right\}\right)$ for $\sigma_{i}, \sigma_{j} \in \widetilde{S}$. Let $s_{i}, s_{j}$ denote the corresponding elements of $S$. One readily sees [Br] that the Coxeter complex $\Sigma\left(\left\{s_{i}, s_{j}\right\}\right)$ is the boundary of a $2 m_{i j}$-gon, where $\operatorname{prod}\left(s_{i}, s_{j}, m_{i j}\right)=\operatorname{prod}\left(s_{j}, s_{i}, m_{i j}\right)$ is the defining relation. Our labelling yields a $2 m_{i j}$-gon $K\left(\left\{\sigma_{i}, \sigma_{j}\right\}\right)$ with left half labelled $\operatorname{prod}\left(\sigma_{i}, \sigma_{j}, m_{i j}\right)$ and right half labelled $\operatorname{prod}\left(\sigma_{j}, \sigma_{i}, m_{i j}\right)$. See Figure 2.1.

We define a quotient $\bar{K}(\tilde{S})$ of $K(\widetilde{S})$ as follows. Identify each vertex of $K(\widetilde{S})$ to a single vertex. From Definition 2.8 each edge has a fixed orientation. Identify each edge labelled $\sigma \in \widetilde{S}$ to a single oriented loop labelled $\sigma$ so that the orientation is preserved. Identify two $p$-cells of $K(\widetilde{S})$ if their boundaries are identified. By Remark 2.10 each 2-cell of $K(\widetilde{S})$ is labelled by a defining relation for $\widetilde{W}(1.3)$. Since the fundamental group is carried by the 2-skeleton we note

Lemma 2.11. $\pi_{1}(\bar{K}(\widetilde{S})) \cong \widetilde{W}$. 
Definition 2.12. $\tilde{K}$ is the universal cover of $\bar{K}(\widetilde{S})$.

Note $\widetilde{K}$ is a union of copies of the fundamental $n$-cell $K(\widetilde{S})$. By Lemma 2.11 the 2-skeleton of $\widetilde{K}$ is the Cayley complex for $\widetilde{W}$. In particular $\widetilde{K}$ is simply connected. In $[\mathrm{T}]$ we show $\widetilde{K}$ is contractible.

\section{POSITIVE WORDS}

A word $P \in F(\widetilde{S})$ is said to be positive if it contains no instance of $\sigma^{-1}$, $\sigma \in \widetilde{S}$. Let $w \in F(S)$ be a geodesic word in $S$. Then the correspondence between $S$ and $\widetilde{S}$ yields a positive word $\tilde{w}$. We call such a word a fundamental

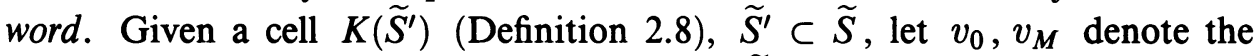
source and sink vertex. Define the diagonal $\Delta\left(\widetilde{S}^{\prime}\right)$ to be any fundamental word labelling an edge path in $K\left(\widetilde{S}^{\prime}\right)$ from $v_{0}$ to $v_{M}$. There are several such words but the ambiguity does not matter here. For example when $\widetilde{S}^{\prime}=\left\{\sigma_{i}, \sigma_{j}\right\}$ we have $\Delta\left({\widetilde{S^{\prime}}}^{\prime}\right)=\operatorname{prod}\left(\sigma_{i}, \sigma_{j}, m_{i j}\right)=\operatorname{prod}\left(\sigma_{j}, \sigma_{i}, m_{i j}\right)(\operatorname{Remark} 2.10)$.

The following preliminary lemmas are due to Garside $[G]$ in the case of the braid group and Brieskorn-Saito [Bri-S] in the general case. Proofs also appear in [D] and [T]. We write $\Delta$ for $\Delta(\widetilde{S})$. We say two words of $F(\widetilde{S})$ are equal if they represent the same element of $\widetilde{W}$.

Lemma 3.1. Any element of $\widetilde{W}$ is represented by a word $\Delta^{-k} P$, where $k \geq 0$ and $P$ is a positive word.

Lemma 3.2. Let $\tilde{w}$ be a fundamental word. Then there exist fundamental words $\tilde{w}_{1}, \tilde{w}_{2}$ such that

(i) $\Delta=\tilde{w}_{1} \tilde{w}$,

(ii) $\Delta \tilde{w}^{\varepsilon} \Delta^{-1}=\tilde{w}_{2}^{\varepsilon}, \varepsilon \in\{-1,+1\}$.

Lemma 3.3. Let $\sigma_{1}, \sigma_{2} \in \tilde{S}$. Let $P_{1}, P_{2}$ be positive words such that $\sigma_{1} P_{1}=$ $\sigma_{2} P_{2}$. Then there exists a positive word $P$ such that $\Delta\left(\left\{\sigma_{1}, \sigma_{2}\right\}\right) P=\sigma_{1} P_{1}$.

The next lemma, and its proof, is adapted from Deligne [D]. If $v, v^{\prime}$ are vertices of $K(\widetilde{S})$ define $d\left(v, v^{\prime}\right)$ to be the length of a minimal length edge path from $v$ to $v^{\prime}$. We use Notation 1.4. After Cannon's notion of cone types [C], we introduce the following partial ordering on vertices of $K(\widetilde{S})$ (Definition 2.8).

Let $v, v^{\prime}$ be vertices of the $n$-cell $K(\widetilde{S})$. Let $v_{0}$ be the source vertex. Define $v^{\prime} \leq v$ if and only if there exists a fundamental word $\tilde{w}$ such that $v=v_{0} \cdot \tilde{w}$ and $[\tilde{w}]_{v_{0}}$ passes through $v^{\prime}$.

Lemma 3.5. Let $v_{0}$ be the source vertex of the $n$-cell $K(\tilde{S})$. Let $B$ be a collection of vertices of $K(\widetilde{S})$ satisfying

(i) $v_{0} \in B$.

(ii) If $v \in B$ and $v^{\prime} \leq v$ then $v^{\prime} \in B$.

(iii) If $z \in B, z \cdot \sigma_{1}, z \cdot \sigma_{2} \in B$ for $\sigma_{1}, \sigma_{2} \in \widetilde{S}$ then $z \cdot \Delta\left(\left\{\sigma_{1}, \sigma_{2}\right\}\right) \in B$.

Then there exists a unique vertex $v_{1} \in B$ such that for each $v \in B, v \leq v_{1}$.

Proof. Uniqueness is clear. Suppose $v \in B$ is distinct from $v_{1}$. Since there is a fundamental word $\tilde{w}$ such that $v_{0} \cdot \tilde{w}=v_{1}$ and $[\tilde{w}]_{v_{0}}$ passes through $v$, we have $d\left(v, v_{0}\right)<d\left(v_{1}, v_{0}\right)$. This implies $v$ cannot satisfy the conclusion. 


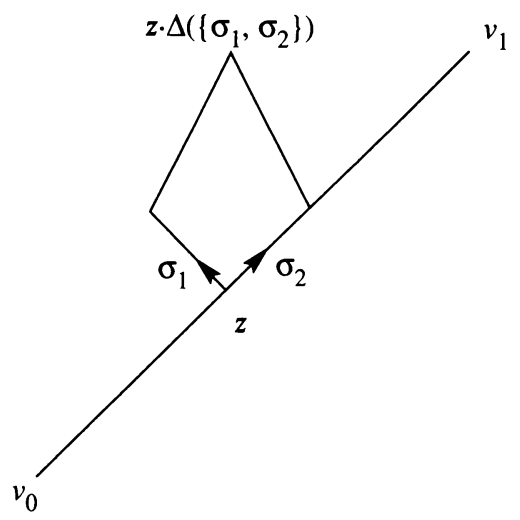

FIGURE 3.1

To prove existence let $v_{1}$ be a vertex of $B$ such that $d\left(v_{1}, v_{0}\right)$ is maximal. It suffices to prove the following assertion. See Figure 3.1.

Let $z \in B, z \leq v_{1}$ and $\sigma_{1}, \sigma_{2} \in \tilde{S}$ such that (a) $z \cdot \sigma_{1}$, (*) $\quad z \cdot \sigma_{2} \in B,(\mathrm{~b}) z \cdot \sigma_{1} \leq v_{1}$ but $z \cdot \sigma_{2}$ is not $\leq v_{1}$. Then there exists $z^{\prime} \in B, z^{\prime} \leq v_{1}$, and $\sigma_{1}^{\prime}, \sigma_{2}^{\prime} \in \widetilde{S}$ such that $z^{\prime}, \sigma_{1}^{\prime}, \sigma_{2}^{\prime}$ satisfy (a), (b) and $d\left(z^{\prime}, v_{0}\right)>d\left(z, v_{0}\right)$.

By (iii) $z \cdot \Delta\left(\left\{\sigma_{1}, \sigma_{2}\right\}\right) \in B$. Since $\Delta\left(\left\{\sigma_{1}, \sigma_{2}\right\}\right)=\operatorname{prod}\left(\sigma_{2}, \sigma_{1}, m_{12}\right)$ we have $z \cdot \sigma_{2} \leq z \cdot \operatorname{prod}\left(\sigma_{2}, \sigma_{1}, m_{12}\right)$. Since $z \cdot \sigma_{2}$ is not $\leq v_{1}$ it follows that $z \cdot$ $\Delta\left(\left\{\sigma_{1}, \sigma_{2}\right\}\right)$ is not $\leq v_{1}$. Let $K\left(\left\{\sigma_{1}, \sigma_{2}\right\}\right)$ denote the 2-cell of $K(\widetilde{S})$ containing $z, z \cdot \sigma_{1}, z \cdot \sigma_{2}$. By examining the edge path of $K\left(\left\{\sigma_{1}, \sigma_{2}\right\}\right)$ traversing $z$, $z \cdot \sigma_{1}, z \cdot \Delta\left(\left\{\sigma_{1}, \sigma_{2}\right\}\right)$ we obtain the desired $z^{\prime}, \sigma_{1}^{\prime}, \sigma_{2}^{\prime}$.

The next proposition is the key ingredient to our solution of the word problem.

Proposition 3.6. Let $\tilde{w}_{L}, \tilde{w}_{R}$ be fundamental words. Then there exist fundamental words $\tilde{x}\left(\tilde{w}_{L}, \tilde{w}_{R}\right), \tilde{u}_{L}, \tilde{u}_{R}$ such that

(i) $\tilde{x}\left(\tilde{w}_{L}, \tilde{w}_{R}\right)=\tilde{w}_{L} \tilde{u}_{L}=\tilde{w}_{R} \tilde{u}_{R}$.

(ii) Whenever $P_{L}, P_{R}$ are positive words such that $\tilde{w}_{L} P_{L}=\tilde{w}_{R} P_{R}$, there exists a positive word $P$ such that $\tilde{x}\left(\tilde{w}_{L}, \tilde{w}_{R}\right) P=\tilde{w}_{L} P_{L}$.

(iii) If a fundamental word $\tilde{x}_{1}\left(\tilde{w}_{L}, \tilde{w}_{R}\right)$ also satisfies (ii) then $\tilde{x}_{1}\left(\tilde{w}_{L}, \tilde{w}_{R}\right)=$ $\tilde{x}\left(\tilde{w}_{L}, \tilde{w}_{R}\right)$.

Before proceeding with the proof we make an observation.

Remark 3.7. Suppose $\tilde{w}_{L}=\sigma_{L}, \tilde{w}_{R}=\sigma_{R}, \sigma_{L}, \sigma_{R}$ distinct elements of $\tilde{S}$. Let $\operatorname{prod}\left(\sigma_{L}, \sigma_{R}, m_{L R}\right)=\operatorname{prod}\left(\sigma_{R}, \sigma_{L}, m_{L R}\right)$ be the defining relation of $\widetilde{W}$ (1.3). By Lemma 3.3 the fundamental words $\tilde{x}\left(\sigma_{L}, \sigma_{R}\right)=\Delta\left(\left\{\sigma_{L}, \sigma_{R}\right\}\right), \tilde{u}_{L}=$ $\operatorname{prod}\left(\sigma_{R}, \sigma_{L}, m_{L R}-1\right), \tilde{u}_{R}=\operatorname{prod}\left(\sigma_{L}, \sigma_{R}, m_{L R}-1\right)$ satisfy the conclusions of Proposition 3.6.

Proof of Proposition 3.6. Recall the complex $\widetilde{K}$ is a union of copies of $K(\widetilde{S})$. Fix one $n$-cell, also denoted $K(\widetilde{S})$, and let $v_{0}$ be its source vertex. Given fundamental words $\tilde{w}_{L}, \tilde{w}_{R}$ we define a set $B$, which depends on $\tilde{w}_{L}, \tilde{w}_{R}$, of vertices of $K(\widetilde{S})$. Note any vertex of the $n$-cell $K(\widetilde{S})$ can be written in the form $v_{0} \cdot \tilde{w}$ for $\tilde{w}$ a fundamental word. 
$v_{0} \cdot \tilde{w} \in B \Leftrightarrow$ whenever $P_{L}, P_{R}$ are positive words such that $\tilde{w}_{L} P_{L}=\tilde{w}_{R} P_{R}$, there exists a positive word $Q$ such that $\tilde{w} Q=$ $\tilde{w}_{L} P_{L}$.

One verifies easily that $B$ satisfies Lemma 3.5(i) and (ii). To verify Lemma 3.5(iii) suppose $z, z \cdot \sigma_{1}, z \cdot \sigma_{2} \in B$ for $\sigma_{1}, \sigma_{2} \in \widetilde{S}$. Let $\tilde{w}$ be a fundamental word such that $z=v_{0} \cdot \tilde{w}$. Suppose $P_{L}, P_{R}$ are positive words such that $\tilde{w}_{L} P_{L}=\tilde{w}_{R} P_{R}$. Since $z \cdot \sigma_{1}, z \cdot \sigma_{2} \in B$ there exists by (3.8) positive words $Q_{1}, Q_{2}$ such that

$$
\tilde{w} \sigma_{1} Q_{1}=\tilde{w} \sigma_{2} Q_{2}=\tilde{w}_{L} P_{L} .
$$

Apply Lemma 3.3. There exists a positive word $Q$ such that

$$
\tilde{w} \Delta\left(\left\{\sigma_{1}, \sigma_{2}\right\}\right) Q=\tilde{w}_{L} P_{L} .
$$

We conclude $v_{0} \cdot \tilde{w} \Delta\left(\left\{\sigma_{1}, \sigma_{2}\right\}\right)=z \cdot \Delta\left(\left\{\sigma_{1}, \sigma_{2}\right\}\right) \in B$, verifying Lemma 3.5(iii). Observe $v_{0} \cdot \tilde{w}_{L}, v_{0} \cdot \tilde{w}_{R} \in B$. Thus by the conclusion of Lemma 3.5 we obtain $\tilde{x}\left(\tilde{w}_{L}, \tilde{w}_{R}\right), \tilde{u}_{L}, \tilde{u}_{R}$ as desired.

Remark 3.9. We keep the notation of the proof of Proposition 3.6. Let $\tilde{w}_{L}$, $\tilde{w}_{R}$ be fundamental words. Let $B$ be the set defined in (3.8) equipped with the partial ordering (3.4). We make the following assertions. Here $\tilde{w}_{1}, \tilde{w}_{2}$ are fundamental words.

(i) Suppose $\tilde{w}_{L}=\sigma \tilde{w}_{L}^{\prime}, \tilde{w}_{R}=\sigma \tilde{w}_{R}^{\prime}$. Then $\tilde{x}\left(\tilde{w}_{L}, \tilde{w}_{R}\right)=\sigma \tilde{x}\left(\tilde{w}_{L}^{\prime}, \tilde{w}_{R}^{\prime}\right)$.

(ii) Let $v_{0} \cdot \tilde{w}_{1} \in B$ and suppose $v_{0} \cdot \tilde{w}_{L} \leq v_{0} \cdot \tilde{w}_{1}$. Then $\tilde{x}\left(\tilde{w}_{1}, \tilde{w}_{R}\right)=$ $\tilde{x}\left(\tilde{w}_{L}, \tilde{w}_{R}\right)$.

(iii) Suppose $v_{0} \cdot \tilde{w}_{1}, v_{0} \cdot \tilde{w}_{2} \in B$. Then $v_{0} \cdot \tilde{x}\left(\tilde{w}_{1}, \tilde{w}_{2}\right) \in B$, hence $v_{0} \cdot$ $\tilde{x}\left(\tilde{w}_{1}, \tilde{w}_{2}\right) \leq v_{0} \cdot \tilde{x}\left(\tilde{w}_{L}, \tilde{w}_{R}\right)$.

Each proof is an exercise in the formal definition of $B$ (3.8). To illustrate we verify (iii). Suppose $P_{L}, P_{R}$ are positive words such that $\tilde{w}_{L} P_{L}=\tilde{w}_{R} P_{R}$. Since $v_{0} \cdot \tilde{w}_{1}, v_{0} \cdot \tilde{w}_{2} \in B$ there exists by (3.8) positive words $Q_{1}, Q_{2}$ such that

$$
\tilde{w}_{1} Q_{1}=\tilde{w}_{2} Q_{2}=\tilde{w}_{L} P_{L} .
$$

By applying Proposition 3.6 to $\tilde{w}_{1}, \tilde{w}_{2}$ there is a fundamental word $\tilde{x}\left(\tilde{w}_{1}, \tilde{w}_{2}\right)$ and a positive word $Q$ such that

$$
\tilde{x}\left(\tilde{w}_{1}, \tilde{w}_{2}\right) Q=\tilde{w}_{1} Q_{1}=\tilde{w}_{l} P_{L} .
$$

By (3.8) $v_{0} \cdot \tilde{x}\left(\tilde{w}_{1}, \tilde{w}_{2}\right) \in B$.

Algorithm 3.10. Let $\tilde{w}_{L}, \tilde{w}_{R}$ be fundamental words. We give an algorithm to find $\tilde{x}\left(\tilde{w}_{L}, \tilde{w}_{R}\right), \tilde{u}_{L}, \tilde{u}_{R}$, the fundamental words given by the conclusion of Proposition 3.6. We reduce the problem of finding $\tilde{x}\left(\tilde{w}_{L}, \tilde{w}_{R}\right)$ to finding $\tilde{x}\left(\tilde{w}_{1}, \tilde{w}_{2}\right)$, where $\left|\tilde{x}\left(\tilde{w}_{1}, \tilde{w}_{2}\right)\right|<\left|\tilde{x}\left(\tilde{w}_{L}, \tilde{w}_{R}\right)\right|$. Write $\tilde{w}_{L}=\sigma_{L} \tilde{w}_{L}^{\prime}, \quad \tilde{w}_{R}=$ $\sigma_{R} \tilde{w}_{R}^{\prime}$. See Figure 3.2.

Case 1. $\sigma_{L}=\sigma_{R}$. Then $\tilde{x}\left(\tilde{w}_{L}, \tilde{w}_{R}\right)=\sigma_{L} \tilde{x}\left(\tilde{w}_{L}^{\prime}, \tilde{w}_{R}^{\prime}\right)$ (Remark 3.9(i)).

Case 2. $\sigma_{L} \neq \sigma_{R}$. We use the notation of Proposition 3.6 and its proof. The set $B$ of vertices of $K(\tilde{S})$ is defined in (3.8). By Lemma 3.3 $v_{0} \cdot \Delta\left(\left\{\sigma_{L}, \sigma_{R}\right\}\right) \in B$. By Remark 3.9(iii) $v_{0} \cdot \tilde{x}\left(\tilde{w}_{L}, \Delta\left(\left\{\sigma_{L}, \sigma_{R}\right\}\right)\right) \in B$ and $\left|\tilde{x}\left(\tilde{w}_{L}, \Delta\left(\left\{\sigma_{L}, \sigma_{R}\right\}\right)\right)\right| \leq$ $\left|\tilde{x}\left(\tilde{w}_{L}, \tilde{w}_{R}\right)\right|$. Since $\Delta\left(\left\{\sigma_{L}, \sigma_{R}\right\}\right)=\sigma_{L} \operatorname{prod}\left(\sigma_{R}, \sigma_{L}, m_{L R}-1\right)$ and $\tilde{w}_{L}=\sigma_{L} \tilde{w}_{L}^{\prime}$

$$
\tilde{x}\left(\tilde{w}_{L}, \Delta\left(\left\{\sigma_{L}, \sigma_{R}\right\}\right)\right)=\sigma_{L} \tilde{x}\left(\tilde{w}_{L}^{\prime}, \operatorname{prod}\left(\sigma_{R}, \sigma_{L}, m_{L R}-1\right)\right) .
$$



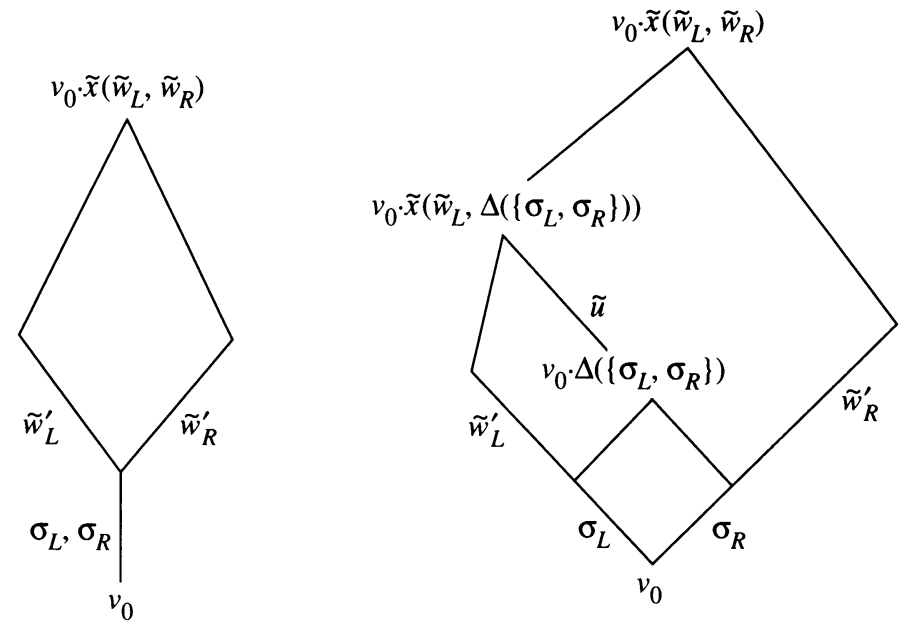

FIGURE 3.2

Since $\left|\tilde{x}\left(\tilde{w}_{L}^{\prime}, \operatorname{prod}\left(\sigma_{R}, \sigma_{L}, m_{L R}-1\right)\right)\right|<\left|\tilde{x}\left(\tilde{w}_{L}, \tilde{w}_{R}\right)\right|$ we can find $\tilde{u}$ such that $\tilde{x}\left(\tilde{w}_{L}^{\prime}, \operatorname{prod}\left(\sigma_{R}, \sigma_{L}, m_{L R}-1\right)\right)=\operatorname{prod}\left(\sigma_{R}, \sigma_{L}, m_{L R}-1\right) \tilde{u}$,

or

$$
\tilde{x}\left(\tilde{w}_{L}, \Delta\left(\left\{\sigma_{L}, \sigma_{R}\right\}\right)\right)=\Delta\left(\left\{\sigma_{L}, \sigma_{R}\right\}\right) \tilde{u} .
$$

Since $v_{0} \cdot \tilde{w}_{L} \leq v_{0} \cdot \Delta\left(\left\{\sigma_{L}, \sigma_{R}\right\}\right) \tilde{u}$, by Remark 3.9(ii)

$$
\begin{aligned}
\tilde{x}\left(\tilde{w}_{L}, \tilde{w}_{R}\right) & =\tilde{x}\left(\Delta\left(\left\{\sigma_{L}, \sigma_{R}\right\}\right) \tilde{u}, \tilde{w}_{R}\right) \\
& =\sigma_{R} \tilde{x}\left(\operatorname{prod}\left(\sigma_{L}, \sigma_{R}, m_{L R}-1\right) \tilde{u}, \tilde{w}_{R}^{\prime}\right) \quad \text { via Remark 3.9(i). }
\end{aligned}
$$

We state a proposition which follows formally from Proposition 3.6 by considering reverse words. Let $P=\sigma_{1} \sigma_{2} \cdots \sigma_{k}$. Define rev $P=\sigma_{k} \cdots \sigma_{2} \sigma_{1}$. One can show (see, for example, [G]) that if $P=Q$ then $\operatorname{rev} P=\operatorname{rev} Q$. We obtain

Proposition 3.11. Let $\tilde{w}_{L}, \tilde{w}_{R}$ be fundamental words. Then there exists fundamental words $\tilde{x}\left(\tilde{w}_{L}, \tilde{w}_{R}\right), \tilde{u}_{L}, \tilde{u}_{R}$ such that

(i) $\tilde{x}\left(\tilde{w}_{L}, \tilde{w}_{R}\right)=\tilde{u}_{L} \tilde{w}_{L}=\tilde{u}_{R} \tilde{w}_{R}$.

(ii) Whenever $P_{L}, P_{R}$ are positive words such that $P_{L} \tilde{w}_{L}=P_{R} \tilde{w}_{R}$, there exists a positive word $P$ such that

$$
P \tilde{x}\left(\tilde{w}_{L}, \tilde{w}_{R}\right)=P_{L} \tilde{w}_{L}
$$

(iii) If a fundamental word $\tilde{x}_{1}\left(\tilde{w}_{L}, \tilde{w}_{R}\right)$ also satisfies (ii) then $\tilde{x}_{1}\left(\tilde{w}_{L}, \tilde{w}_{R}\right)=$ $\tilde{x}\left(\tilde{w}_{L}, \tilde{w}_{R}\right)$.

Remark 3.12. Given $\tilde{w}_{L}, \tilde{w}_{R}$ one can find $\tilde{x}\left(\tilde{w}_{L}, \tilde{w}_{R}\right)$ satisfying Proposition 3.11 by applying Algorithm 3.10 to $\operatorname{rev} \tilde{w}_{L}, \operatorname{rev} \tilde{w}_{R}$. Alternatively one can reformulate Algorithm 3.10. Some examples are indicated in Figures 4.3, 4.4.

\section{THE ISOPERIMETRIC INEQUALITY}

In this section we show $\widetilde{W}$ satisfies a quadratic isoperimetric inequality, describe an explicit solution to the word problem in quadratic time, and give two specific examples. 
Fix a vertex $v_{0}$ of $\widetilde{K}$ (Definition 2.12). Define a cell $K^{\prime}$ of $\widetilde{K}$ to be positive if for each vertex $v$ of $K^{\prime}$ there is a positive word $P$ such that $v=v_{0} \cdot P$ (Notation 1.4). Define

$$
\widetilde{K}_{+}=\bigcup_{K^{\prime} \text { positive }} K^{\prime} .
$$

Given $m \geq 0$ define $\left(\widetilde{K}_{+}\right)_{m}$ to be the union of all positive cells $K^{\prime}$ such that each vertex $v_{0} \cdot P$ of $K^{\prime}$ satisfies $|P| \leq m$. We refer the reader to [CEHPT] for a geometric interpretation of the quadratic isoperimetric inequality. We verify the quadratic isoperimetric inequality by contracting closed edge paths in $\widetilde{K}_{+}$. However the length associated to such a path is given by a metric not equal to but quasi-isometric with the standard edge path metric.

Definition 4.1. $C=\{\tilde{w}: \tilde{w}$ is a fundamental word $\}$.

We use Proposition 3.11 to define a set of relations.

Definition 4.2. $R=\left\{\tilde{u}_{L} \tilde{w}_{L}=\tilde{u}_{R} \tilde{w}_{R}: \tilde{u}_{L}, \tilde{u}_{R} \in C\right.$ are obtained by applying Proposition 3.11 to $\left.\tilde{w}_{L}, \tilde{w}_{R} \in C\right\}$.

Since $\tilde{S} \subset C, C$ is a generating set. Since $C$ is finite the metric induced on $\widetilde{K}$ by $C$ is quasi-isometric to the edge path metric. An edge path $T$ in $\widetilde{K}$ is a $C$-parametrized path if associated to $T$ there is $f \in F(C)$ and a vertex $v \in T$ such that $T=[f]_{v}$. We say $f$ parametrizes $T$. We define the $C$-length of $T$ (or $f$ ) to be the length of $f$ as a word in $C$. We let $|f|_{C}$ denote the $C$-length of $f$.

Let $T=[f]_{v}$ be a $C$-parametrized path parametrized by $f \in F(C)$. Suppose $f^{\prime} \in F(C)$ is obtained from $f$ by a single application of a relator $\tilde{u}_{L} \tilde{w}_{L}=\tilde{u}_{R} \tilde{w}_{R} \in R$. We say $f^{\prime}$ is obtained from $f$ by an $R$-substitution and the $C$-parametrized path $\left[f^{\prime}\right]_{v}$ is obtained from $T$ by an $R$-homotopy (Figure 4.1). Since $\tilde{u}_{L} \tilde{w}_{L}=\tilde{u}_{R} \tilde{w}_{R}$ is a fundamental word (Proposition 3.11) the relation labels an edge path on $\partial K(\widetilde{S})$, where $K(\widetilde{S})$ is some $n$-cell of $\widetilde{K}$. We can then perform the $R$-homotopy across $K(\widetilde{S})$. This implies an $R$-homotopy is cellular.

We make a preliminary observation.

Remark 4.3. Let $f=\tilde{w}_{1}^{\varepsilon_{1}} \tilde{w}_{2}^{\varepsilon_{2}} \cdots \tilde{w}_{p}^{\varepsilon_{p}} \in F(C), \varepsilon_{i} \in\{-1,+1\}$. Then $[f]_{v_{0} \cdot \Delta^{p}} \subset$ $\widetilde{K}_{+}$. To see this write $f_{j}=\widetilde{w}_{1}^{\varepsilon_{1}} \cdots \widetilde{w}_{j}^{\varepsilon_{j}}, 1 \leq j \leq p$. By repeated use of Lemma 3.2(ii) we can find $\tilde{u}_{1}, \ldots, \tilde{u}_{j}$ such that

$$
\Delta^{p} f_{j}=\Delta^{p-j} \Delta^{j} \tilde{w}_{1}^{\varepsilon_{1}} \cdots \tilde{w}_{j}^{\varepsilon_{j}}=\Delta^{p-j} \Delta \tilde{u}_{1}^{\varepsilon_{1}} \cdots \Delta \tilde{u}_{j}^{\varepsilon_{j}} .
$$

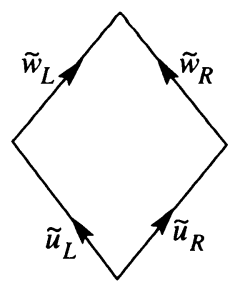

FIGURE 4.1. $R$-homotopy 
By Lemma 3.2(i) each $\Delta \tilde{u}_{i}^{\varepsilon_{i}}$ equals a positive word, hence $v_{0} \cdot \Delta^{p} f_{j} \in \tilde{K}_{+}$for each $j$. It follows that $[f]_{v_{0} \cdot \Delta^{p}}$ remains in $\widetilde{K}_{+}$.

Theorem 4.4 (Quadratic Isoperimetric Inequality). Suppose $T$ is a $C$-parametrized closed path in $\widetilde{K}$ of $C$-length $p$. Then at most $2|\Delta| \cdot p^{2} \quad R$-homotopies are needed to contract $T$ to a single vertex.

Proof. Let $f=\tilde{w}_{1}^{\varepsilon_{1}} \tilde{w}_{2}^{\varepsilon_{2}} \cdots \tilde{w}_{p}^{\varepsilon_{p}} \in F(C), \varepsilon_{i} \in\{-1,+1\}$, parametrize $T$. We may assume $T=[f]_{v_{0} \cdot \Delta^{p}}$. By Remark $4.3 T \subset \widetilde{K}_{+}$. Set

$$
M=\inf \left\{m: T \subset\left(\widetilde{K}_{+}\right)_{m}\right\} .
$$

Each $\tilde{w} \in C$ satisfies $|\tilde{w}| \leq|\Delta|$. Thus $|f| \leq|\Delta| \cdot p$. Then $M \leq\left|\Delta^{p}\right|+|f| \leq$ $2|\Delta| \cdot p$.

Set $f_{j}=\tilde{w}_{1}^{\varepsilon_{1}} \tilde{w}_{2}^{\varepsilon_{2}} \cdots \tilde{w}_{j}^{\varepsilon_{j}}$. There exists $j, 1 \leq j \leq p$, such that $v=v_{0} \cdot \Delta^{p} f_{j} \in$ $\left(\widetilde{K}_{+}\right)_{M} \backslash\left(\widetilde{K}_{+}\right)_{M-1}$. Note there are at most $p$ such $j$. If $j<p$ set $\tilde{w}_{L}=\tilde{w}_{j}^{\varepsilon_{j}}$, $\tilde{w}_{R}=\tilde{w}_{j+1}$. By the definition of $M, \varepsilon_{j}=+1, \varepsilon_{j+1}=-1$. If $j=p$ set $\tilde{w}_{L}=\tilde{w}_{P}^{\varepsilon_{p}}, \tilde{w}_{R}=\tilde{w}_{1}$. Then $\varepsilon_{p}=+1, \varepsilon_{1}=-1$.

Since $T \subset \widetilde{K}_{+}, v, v \cdot\left(\tilde{w}_{L}\right)^{-1}, v \cdot\left(\tilde{w}_{R}\right)^{-1} \in \widetilde{K}_{+}$. By Proposition 3.11 we can find (possibly trivial) $\tilde{u}_{L}, \tilde{u}_{R} \in C$ such that $\tilde{u}_{L} \tilde{w}_{L}=\tilde{u}_{R} \tilde{w}_{R}$ and $v \cdot\left(\tilde{u}_{L} \tilde{w}_{L}\right)^{-1} \in$ $\left(\tilde{K}_{+}\right)_{M-1}$. Let $f_{1} \in F(C)$ denote the word obtained from $f$ by replacing $\tilde{w}_{L}\left(\tilde{w}_{R}\right)^{-1}$ by $\left(\tilde{u}_{L}\right)^{-1} \tilde{u}_{R}$, and let $T_{1}$ denote the corresponding $C$-parametrized path. Then $T_{1} \subset \widetilde{K}_{+}$and $\left|f_{1}\right|_{C} \leq|f|_{C}$.

At most $p$ such $R$-homotopies are needed to contract $T=[f]_{v_{0} \cdot \Delta^{p}}$ to a $C$-parametrized path $T^{\prime}=\left[f^{\prime}\right]_{v}, f^{\prime} \in F(C)$ and $v$ a vertex of $T^{\prime}$, such that

$$
\left|f^{\prime}\right|_{C} \leq|f|_{C}, \quad\left[f^{\prime}\right]_{v} \subset\left(\widetilde{K}_{+}\right)_{M-1} .
$$

It follows that the number of $R$-homotopies needed to contract $T$ to $v_{0}$ is at most $p \cdot M \leq 2|\Delta| \cdot p^{2}$.

We turn now to the word problem. Our algorithm applies to words $f \in$ $F(C)$. Let $f=\tilde{w}_{1}^{\varepsilon_{1}} \tilde{w}_{2}^{\varepsilon_{2}} \cdots \tilde{w}_{p}^{\varepsilon_{p}} \in F(C), \varepsilon_{i} \in\{-1,+1\}$. Write $f_{j}=\tilde{w}_{1}^{\varepsilon_{1}} \tilde{w}_{2}^{\varepsilon_{2}} \cdots$ $\tilde{w}_{j}^{\varepsilon_{j}}, 1 \leq j \leq p$. Set $v_{j}(f)=v_{0} \cdot f_{j}$. We say $f$ satisfies Property 4.5 if

Property 4.5. $v_{i}(f) \in \widetilde{K}_{+} \Rightarrow v_{j}(f) \in \widetilde{K}_{+}$for each $j \leq i$.

Lemma 4.6. Suppose $f=\tilde{w}_{1}^{\varepsilon_{1}} \tilde{w}_{2}^{\varepsilon_{2}} \cdots \tilde{w}_{p}^{\varepsilon_{p}} \in F(C)$ satisfies Property 4.5. Suppose $\varepsilon_{i}=+1$ and $\varepsilon_{i+1}=-1$, some $i$ such that $1 \leq i<p$. Let $f^{\prime} \in F(C)$ be obtained from $f$ by the R-substitution $\left(\tilde{u}_{i}\right)^{-1} \tilde{u}_{i+1}$ for $\tilde{w}_{i}\left(\tilde{w}_{i+1}\right)^{-1}$, where $\tilde{u}_{i} \tilde{w}_{i}=\tilde{u}_{i+1} \tilde{w}_{i+1} \in R$. Then $f^{\prime}$ satisfies Property 4.5.

Proof. Set $v_{i}^{\prime}=v_{i-1} \cdot\left(\tilde{u}_{i}\right)^{-1}$. If either $v_{i}^{\prime}=v_{i-1}$ or $v_{i}^{\prime}=v_{i+1}$ then Property 4.5 clearly continues to hold for $f^{\prime}$ (see Figure 4.2). We may assume $v_{i}^{\prime}$ is distinct from $v_{i-1}, v_{i+1}$. There are two cases. Suppose $v_{1}^{\prime} \in \widetilde{K}_{+}$. Then $v_{i-1}=v_{i}^{\prime} \cdot \tilde{u}_{i} \in \widetilde{K}_{+}$. Since $f$ satisfies Property $4.5 v_{j} \in \widetilde{K}_{+}$for each $j \leq i-1$. Thus $f^{\prime}$ satisfies Property 4.5. Suppose $v_{1}^{\prime} \notin \widetilde{K}_{+}$and there exists $j \geq i+1$ such that $v_{j} \in \widetilde{K}_{+}$. Since $f$ satisfies Property $4.5 v_{i-1}, v_{i}, v_{i+1} \in \widetilde{K}_{+}$. But by the definition of $R$ this implies $v_{i}^{\prime} \in \widetilde{K}_{+}$, a contradiction. We conclude $f^{\prime}$ satisfies Property 4.5. 

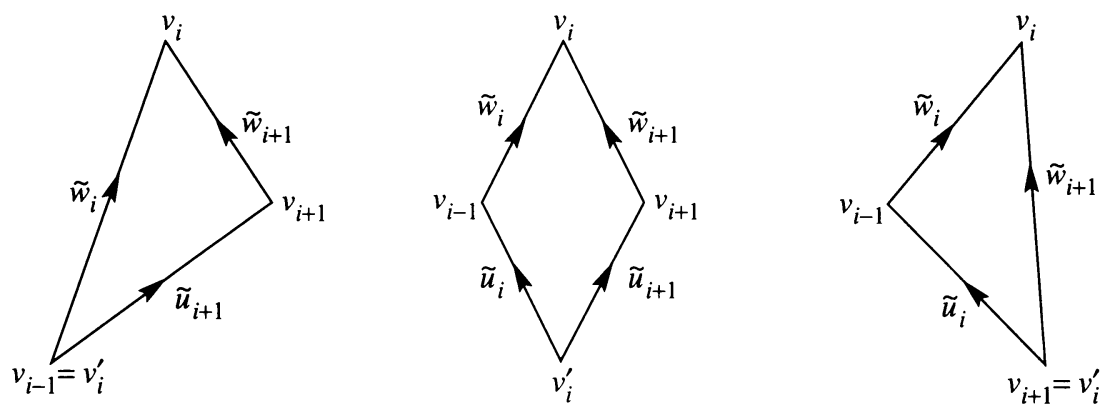

FIGURE 4.2

There is a map $e: F(\widetilde{S}) \rightarrow Z$ given by the exponent sum. Let $f=$ $\tilde{w}_{1}^{\varepsilon_{1}} \tilde{w}_{2}^{\varepsilon_{2}} \cdots \tilde{w}_{p}^{\varepsilon_{p}} \in F(C)$. Define

$$
\begin{gathered}
J(f)=\left\{i, 0<i<p: \varepsilon_{i}=+1, \varepsilon_{i+1}=-1\right\} . \\
M(f)= \begin{cases}0 & \text { if } J(f)=\varnothing, \\
\sup _{i \in J} e\left(\tilde{w}_{1}^{\varepsilon_{1}} \tilde{w}_{2}^{\varepsilon_{2}} \cdots \tilde{w}_{i}^{\varepsilon_{i}}\right) \quad \text { if } J(f) \neq \varnothing .\end{cases}
\end{gathered}
$$

In order to solve the word problem it suffices, by Lemma 3.1, to decide when two positive words are equal. Let $P, Q$ be nontrivial positive words. We apply our algorithm to words of the form $P Q^{-1}=\tilde{w}_{1} \cdots \tilde{w}_{k}\left(\tilde{w}_{k+1}\right)^{-1} \cdots\left(\tilde{w}_{p}\right)^{-1}$. The algorithm is contained in the proof of the following.

Theorem 4.8 (Algorithm to Solve the Word Problem). Let $f=\tilde{w}_{1} \cdots \tilde{w}_{k}\left(\tilde{w}_{k+1}\right)^{-1}$ $\cdots\left(\tilde{w}_{p}\right)^{-1} \in F(C)$. Then at most $|\Delta| \cdot p^{2} \quad R$-substitutions are needed to decide if $f$ represents the identity.

Proof. Note $f$ satisfies Property 4.5 and $0<M(f)<p \cdot|\Delta|$. The algorithm consists of applying the following reduction step to $f$ and iterating the procedure as often as possible. The procedure reduces $M(f)$.

Reduction step. Let $g=\tilde{z}_{1}^{\varepsilon_{1}} \tilde{z}_{2}^{\varepsilon_{2}} \cdots \tilde{z}_{q}^{\varepsilon_{q}}$, where $\tilde{z}_{i} \in C$ and $\varepsilon_{i} \in\{-1,+1\}$. Suppose $\varepsilon_{1}=+1$, and there exists $\varepsilon_{i}=-1$. Then $M(g)>0$. We define the reduction $g^{\prime} \in F(C)$ of $g$ as follows. Set

$$
I=\left\{i \in J(g): e\left(\tilde{z}_{1}^{\varepsilon_{1}} \tilde{z}_{2}^{\varepsilon_{2}} \cdots \tilde{z}_{i}^{\varepsilon_{i}}\right)=M(g)\right\} .
$$

Then for each $i \in I$ there exists $\tilde{u}_{i}, \tilde{u}_{i+1} \in C$ such that

$$
\tilde{u}_{i} \tilde{z}_{i}=\tilde{u}_{i+1} \tilde{z}_{i+1} \in R \text {. }
$$

Let $g^{\prime}$ be the word obtained from $g$ via the $R$-substitutions $\left(\tilde{u}_{i}\right)^{-1} \tilde{u}_{i+1}$ for $\tilde{z}_{i}\left(\tilde{z}_{i+1}\right)^{-1}$ for each $i \in I$. Note at most $|g|_{C} R$-substitutions are used. Further

$$
\left|g^{\prime}\right|_{C} \leq|g|_{C}, \quad M\left(g^{\prime}\right)<M(g) .
$$

We eventually obtain a word $f^{\prime}=\tilde{x}_{1}^{\varepsilon_{1}} \tilde{x}_{2}^{\varepsilon_{2}} \ldots \tilde{x}_{r}^{\varepsilon_{r}} \in F(C)$ such that the reduction step does not apply. Since we only apply the reduction step to a word $g$ with $M(g)>0$, and the resulting word $g^{\prime}$ satisfies $M\left(g^{\prime}\right)<M(g)$, we obtain $f^{\prime}$ after at most $M(f)$ reductions steps. Recall $p=|f|_{C}$. Since each reduction step does not increase the $C$-length, at most $M(f) \cdot p \leq|\Delta| \cdot p^{2}$ $R$-substitutions are needed to transform $f$ to $f^{\prime}$. 


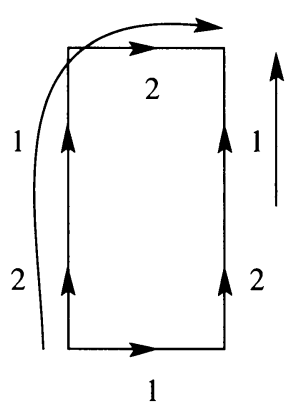

$212 \cdot 1^{-}=12$

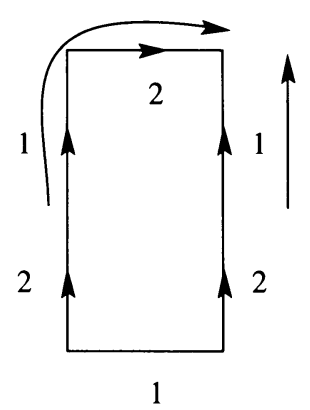

$12 \cdot 1^{-}=2^{-} \cdot 12$

FIGURE 4.3

Since we cannot apply the reduction step to $f^{\prime}$ either $f^{\prime}$ is the trivial word, $\varepsilon_{1}=-1$, or $\varepsilon_{i}=+1$ for each $i$. If $\varepsilon_{1}=-1$ then $v_{0} \cdot \tilde{x}_{1}^{-1} \notin \widetilde{K}_{+}$. Since $f$ satisfies Property 4.5 , by Lemma 4.6 so does $f^{\prime}$. Hence $v_{0} \cdot f^{\prime}=v_{0} \cdot f \notin \widetilde{K}_{+}$. We conclude $f$ is not equal to a positive word. If each $\varepsilon_{i}=+1$ we conclude $f=f^{\prime}$ is a nontrivial positive word.

We give two explicit examples of the algorithm to solve the word problem. We emphasize that the relations used to reduce the words are those given by Proposition 3.11. We also replace $(\tilde{u} \tilde{w})^{\varepsilon}$ with $(\tilde{x})^{\varepsilon}$, where $\tilde{u}, \tilde{w}, \tilde{x} \in C$, $\tilde{x}=\tilde{u} \tilde{w}$, and $\varepsilon \in\{-1,+1\}$. This substitution strictly reduces $C$-length.

Example 4.9. $\widetilde{W}=B_{3}=\left\langle\sigma_{1}, \sigma_{2}: \sigma_{1} \sigma_{2} \sigma_{1}=\sigma_{2} \sigma_{1} \sigma_{2}\right\rangle$.

Let $P=\sigma_{2} \sigma_{1}^{4} \sigma_{2} \sigma_{1} \sigma_{2}^{3} \sigma_{1} \sigma_{2}, Q=\sigma_{1}^{2} \sigma_{2} \sigma_{1}^{2} \sigma_{2}^{2} \sigma_{1}^{5}$. The symbol $i$ represents $\sigma_{i}$. We decompose $P Q^{-1}$ as a product of fundamental words and their inverses. $P Q^{-1}=21 \cdot 1 \cdot 1 \cdot 121 \cdot 2 \cdot 2 \cdot 212 \cdot 1^{-} \cdot 1^{-} \cdot 1^{-} \cdot 1^{-} \cdot 1^{-} 2^{-} \cdot 2^{-} 1^{-} \cdot 1^{-} 2^{-} 1^{-} \cdot 1^{-}$.

We examine $212 \cdot 1^{-}$. See Figure 4.3. We have $212 \cdot 1^{-}=12$. Then

$$
P Q^{-1}=21 \cdot 1 \cdot 1 \cdot 121 \cdot 2 \cdot 2 \cdot 12 \cdot 1^{-} \cdot 1^{-} \cdot 1^{-} \cdot 1^{-} 2^{-} \cdot 2^{-} 1^{-} \cdot 1^{-} 2^{-} 1^{-} \cdot 1^{-} \text {. }
$$

We examine $12 \cdot 1^{-}$. We use the relation $12 \cdot 1^{-}=2^{-} \cdot 12$. Then

$$
\begin{aligned}
P Q^{-1} & =21 \cdot 1 \cdot 1 \cdot 121 \cdot 2 \cdot \mathbf{2} \cdot 2^{-} \cdot 12 \cdot 1^{-} \cdot 1^{-} \cdot 1^{-} 2^{-} \cdot 2^{-} 1^{-} \cdot 1^{-} 2^{-} 1^{-} \cdot 1^{-} \\
& =21 \cdot 1 \cdot 1 \cdot 121 \cdot 2 \cdot 12 \cdot 1^{-} \cdot 1^{-} \cdot 1^{-} 2^{-} \cdot 2^{-} 1^{-} \cdot 1^{-} 2^{-} 1^{-} \cdot 1^{-} \\
& =21 \cdot 1 \cdot 1 \cdot 121 \cdot 2 \cdot 2^{-} \cdot 12 \cdot 1^{-} \cdot 1^{-} 2^{-} \cdot 2^{-} 1^{-} \cdot 1^{-} 2^{-} 1^{-} \cdot 1^{-} \\
& =21 \cdot 1 \cdot 1 \cdot 121 \cdot 12 \cdot 1^{-} \cdot 1^{-} 2^{-} \cdot 2^{-} 1^{-} \cdot 1^{-} 2^{-} 1^{-} \cdot 1^{-} \\
& =21 \cdot 1 \cdot 1 \cdot 121 \cdot 2^{-} \cdot 12 \cdot 1^{-} 2^{-} \cdot 2^{-} 1^{-} \cdot 1^{-} 2^{-} 1^{-} \cdot 1^{-} \\
& =21 \cdot 1 \cdot 1 \cdot \mathbf{1 2 1} \cdot 2^{-} \cdot 2^{-} \cdot 1 \cdot 2^{-} 1^{-} \cdot 1^{-} 2^{-} 1^{-} \cdot 1^{-} \\
& =21 \cdot 1 \cdot 1 \cdot 21 \cdot 2^{-} \cdot \mathbf{1} \cdot 2^{-} 1^{-} \cdot 1^{-} 2^{-} 1^{-} \cdot 1^{-} \\
& =21 \cdot 1 \cdot 1 \cdot 1^{-} \cdot \mathbf{2 1} \cdot 2^{-} 1^{-} \cdot 2 \cdot 1^{-} 2^{-} 1^{-} \cdot 1^{-} \\
& =21 \cdot 1 \cdot \mathbf{1} \cdot \mathbf{1}^{-} \cdot 1^{-} \cdot 2 \cdot 2 \cdot \mathbf{1}^{-} 2^{-} 1^{-} \cdot 1^{-} \\
& =21 \cdot \mathbf{1} \cdot \mathbf{1}^{-} \cdot \mathbf{2} \cdot \mathbf{1}^{-} \mathbf{2}^{-} \cdot 1^{-} \\
& =\mathbf{2 1} \cdot \mathbf{1}^{-} \mathbf{2}^{-} \cdot 1 \cdot \mathbf{1}^{-} \\
& =e .
\end{aligned}
$$




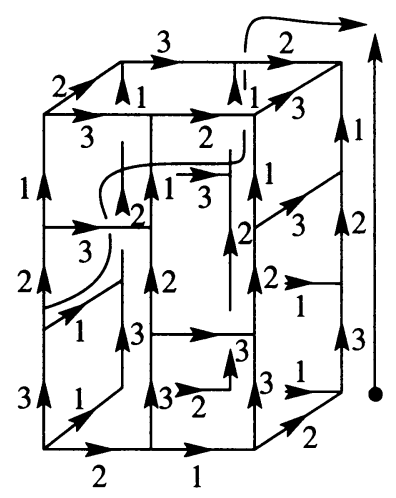

$12312 \cdot 1^{-2}-3=3^{-} \cdot 212$

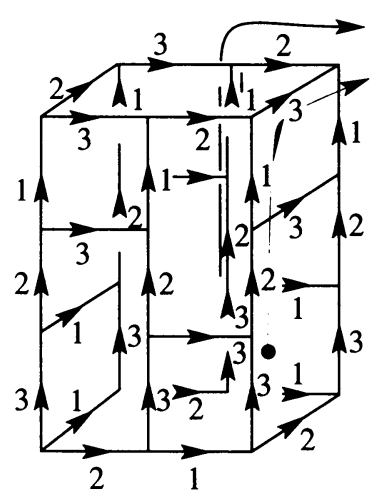

$212 \cdot 3^{-} 1^{-2}=3^{-} 2^{-} 1^{-} \cdot 213$

FIGURE 4.4

Example 4.10. $\widetilde{W}=B_{4}=\left\langle\sigma_{1}, \sigma_{2}, \sigma_{3}: \sigma_{1} \sigma_{2} \sigma_{1}=\sigma_{2} \sigma_{1} \sigma_{2}, \sigma_{2} \sigma_{3} \sigma_{2}=\sigma_{3} \sigma_{2} \sigma_{3}, \sigma_{1} \sigma_{3}\right.$ $=\sigma_{3} \sigma_{1}$. .

We write $i$ for $\sigma_{i}$. Let $P=3^{2} 1232^{2} 1^{2} 21, Q=213^{2} 21^{5} 2$. We decompose $P Q^{-1}$ as a product of fundamental words and their inverses. See Figure 4.4. Then

$$
\begin{aligned}
P Q^{-1} & =3 \cdot 3123 \cdot 2 \cdot 21 \cdot 121 \cdot 2^{-} 1^{-} \cdot 1^{-} \cdot 1^{-} \cdot 1^{-} \cdot 1^{-} 2^{-} 3^{-} \cdot 3^{-} 1^{-} 2^{-} \\
& =3 \cdot 3123 \cdot 2 \cdot 21 \cdot 2 \cdot 1^{-} \cdot 1^{-} \cdot 1^{-} \cdot 1^{-} 2^{-} 3^{-} \cdot 3^{-} 1^{-} 2^{-} \\
& =3 \cdot 3123 \cdot 2 \cdot 212 \cdot 1^{-} \cdot 1^{-} \cdot 1^{-} \cdot 1^{-} 2^{-} 3^{-} \cdot 3^{-} 1^{-} 2^{-} \\
& =3 \cdot 3123 \cdot 2 \cdot 12 \cdot 1^{-} \cdot 1^{-} \cdot 1^{-} 2^{-} 3^{-} \cdot 3^{-} 1^{-} 2^{-} \\
& =3 \cdot 3123 \cdot 212 \cdot 1^{-} \cdot 1^{-} \cdot 1^{-} 2^{-} 3^{-} \cdot 3^{-} 1^{-} 2^{-} \\
& =3 \cdot 3123 \cdot 12 \cdot 1^{-} \cdot 1^{-} 2^{-} 3^{-} \cdot 3^{-} 1^{-} 2^{-} \\
& =3 \cdot 312312 \cdot 1^{-} \cdot 1^{-} 2^{-} 3^{-} \cdot 3^{-} 1^{-} 2^{-} \\
& =3 \cdot 12312 \cdot 1^{-} 2^{-} 3^{-} \cdot 3^{-} 1^{-} 2^{-} \\
& =3 \cdot 3^{-} \cdot 212 \cdot 3^{-} 1^{-} 2^{-} \\
& =212 \cdot 3^{-} 1^{-} 2^{-} \\
& =3^{-} 2^{-} 1^{-} \cdot 213 .
\end{aligned}
$$

We conclude $P Q^{-1}$ is neither the identity nor equal to a positive word.

\section{REFERENCES}

[Bi] J. Birman, Braids, links, and mapping class groups, Ann. of Math. Stud., no. 82, Princeton Univ. Press, Princeton, N.J., 1974.

[Bri-S] E. Brieskorn and K. Saito, Artin-Gruppen und Coxeter-Gruppen, Invent. Math. 17 (1972).

[Br] K. S. Brown, Buildings, Springer-Verlag, New York, 1989.

[C] J. W. Cannon, The combinatorial structure of cocompact discrete hyperbolic groups, Geom. Dedicata 16 (1983), 123-148.

[Ch] R. Charney, (to appear).

[CEHPT] J. W. Cannon, D. B. A. Epstein, D. F. Holt, M. S. Paterson, and W. P. Thurston, Word processing and group theory. 
[Da] M. W. Davis, Groups generated by reflections and aspherical manifolds not covered by Euclidean space, Ann. of Math. (2) 117 (1983).

[D] P. Deligne, Les immeubles des groupes de tresses généralisés, Invent. Math. 17 (1972), 273-302.

[G] F. A. Garside, The braid groups and other groups, Quart. J. Math. Oxford Ser. (2) 20 (1969), 235-254.

[Ge-S] S. Gersten and H. Short, Small cancellation theory and automatic groups, Invent. Math. 102 (1990), 305-334.

[Gr] M. Gromov (S. Gersten, ed.), Essays in group theory, MSRI publication, Springer-Verlag, 1987.

[Sq] C. Squier, The homological algebra of Artin groups, preprint.

[T] K. Tatsuoka, $A$ finite $K(\pi, 1)$ for Artin groups of finite type, preprint.

[Th] W. Thurston, Finite state algorithms for the braid groups, preprint.

Current address: 24 W. 2nd St., \#6, Corning, New York 14830 